\title{
TELEMETRIC SYSTEM OF SIX DEGREES OF FREEDOM MANIPULATOR
}

\begin{abstract}
A. Pilis ${ }^{*}$, M. Rećko ${ }^{* *}$, K. Dzierżek ${ }^{* * *}$
Abstract: The purpose of the following project is to implement the telemetric system of six-axis mode manipulator with six rotary joints. The telemetric system will be an integral part of operator's control application, used for controlling the arm mounted on the driving platform of the remotely controlled robot. The primary objective of the system is to help the robot's through displaying actual position of each joint on the CAD model in 3D. The first step of system design is the creation of the CAD model of the robot's arm, with consideration of mount places for angle position measuring devices for each degree of freedom. The next part of work is the design of PCB circuitry. Another step is to make the physical model of the manipulator and testing the correctness of generated data. In the next step, it is necessary to write the program in programming language allowing to animate the robotic arm motion using the data received from the robot, e.g. $C++$ language cooperating with OpenGL software. Thanks to this solution task entrusted to the operator may be done more accurately, and also help to find the source of occurring errors.
\end{abstract}

Keywords: telemetric system, telemetric, OpenGL, angle position

\section{Introduction}

Telemetry is a technical and scientific domain used for wireless inspection of media usage (Cruz, 2017). It enables decrease amount of workers needed to control the accuracy of the ongoing process. The telemetry system is a connection of device set consisting of the measurement system. It's designed to collect observed parameters, send it to a central device, and analyse incoming data. Received data can be used to check system run.

Robotics is science domain still in a development phase. Telemetry may be used to control as many work parameters of manipulation device set as user needs. An example of telemetry, used in robotics, may be inverse kinematics of remote-controlled robot's or industry robot's manipulator. As inverse kinematics, we understand steps leading to finding generalised positions and forces of each joint (Kozlowski, 2003). To calculate the inverse kinematics of robotic arm we need to know arm's spatial location. In purpose of that, it is necessary to find numerous of position variables (Craig, 1989).

\section{The robotic arm}

Telemetric system was specially created to use with RED Mars Rover Analogue's six-axis manipulator. All arm joints are rotational. The first part of the arm - "turntable", is rotating in a horizontal plane (Fig. 1a). Next, a chain of three joints (b, c, d), turning in a vertical plane is used. Fifth rotation axis (e) is located perpendicular to previous joints, at a rotation axis of the gripper. Last part of the arm is a gripper, powered by screw allowing fingers to open and close (f).

Adrian Pilis, Eng., Bialystok University of Technology, Faculty of Mechanical Engineering, Wiejska 45C; 15-351, Białystok; PL, adrianpilis@gmail.com

** Maciej Rećko, MSc Eng., Bialystok University of Technology, Faculty of Mechanical Engineering, Wiejska 45C; 15-351, Białystok; PL, m.recko@outlook.com

**** Kazimierz Dzierżek, PhD Eng., Bialystok University of Technology, Faculty of Mechanical Engineering, Wiejska 45C; 15351, Białystok; PL, k.dzierzek@pb.edu.pl 


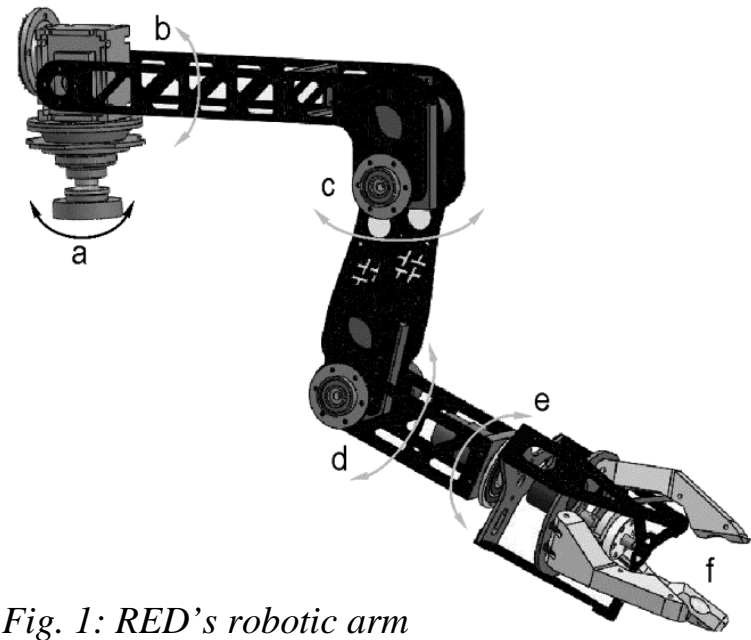

Consideration of telemetry system started with making a 3D model of the manipulator. To do so, we needed to think how we want our manipulator to be represented in a virtual environment (SolidWorks, 2017 and Sydor, 2009). 3D CAD models were used. It had to have the same dimensions as an arm to allow precise calculation of each joint's movement.

In the beginning, the real-life range of movement of the manipulator was measured. It is naturally restricted by mechanical bonds and wiring. When it was done, we estimated measuring the spectrum of arm's movement restricting them to safe scope.

\section{Telemetry system design}

As a result of kinematics structure analysis, maximal angles of relative rotation of each link was obtained. Then, encoders were chosen accordingly to the measured range and considering the possibility of their placement on the axes.
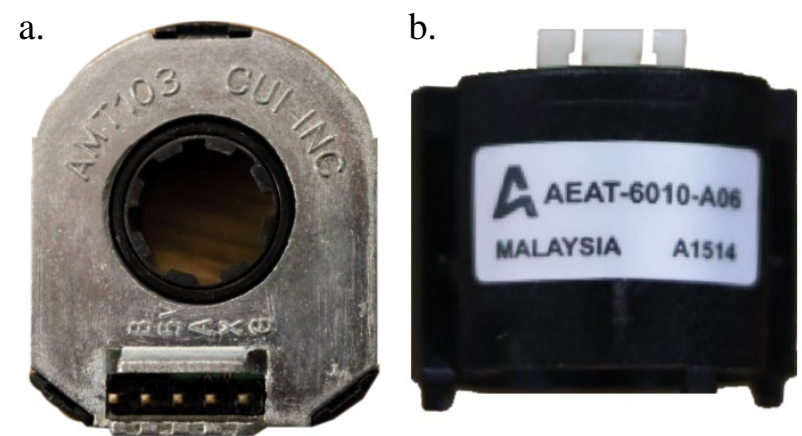

Fig. 2: Magnetic encoder: $a$. incremental; $b$. absolute

We have decided to use magnetic absolute encoders - AEAT-6010-A06- located at four out of six rotation axes (rotation points a-d, Fig. 1) and showed in Fig. 2a. We have chosen magnetic absolute encoders because of its position memory allowing to find out arm's absolute position of every joint (Dzierzek, 2009). Absolute encoders are utilising the Hall effect to measure angle position. Magnetic construction provides the ability of touchless operation, contributing to the long lifespan of the encoder. One used in the rover can detect rotations of $0.35^{\circ}$. YT2010-28 incremental encoder is used to measure the rotation of the gripper. It is integrated with a motor's shaft. The problem that occurred with the use of this encoder was the data format that the device outputted. To interpret the information as an angle of gripper's rotation, we had to include in our calculations the motor's ratio.

Final encoder used in our telemetric system is incremental encoder: AMT103-V (Fig. 2b). It measures the distance between gripper's fingers indirectly. The motor powered trapezoidal screw gear that propelled fingers of the end effector. The formula used to calculate finger's position had to include the coefficient of conversion (turns into the distance), obtained through the experiment.

\section{Electronic components}

Next step of building telemetry system is modelling custom PCB circuit with STM microcontroller onboard. This approach allowed us to use only inputs and outputs we need. We may also change the purpose of most pins in the microcontroller.

All encoders use the same power line. AEAT encoders use the same line for the clock signal and index signal. Power for all encoders is coming from one source, so there is no problem with the different voltage on encoders.

Data read by PCB board is transmitted to a PC by means of Satteline radio modems. The connection provided is transparent on both ends and allows remote access to the serial port of designed solution. The PC has to decode received data and interpret readings as angles used for visualisation of the telemetered robotic arm (Fig. 3). 


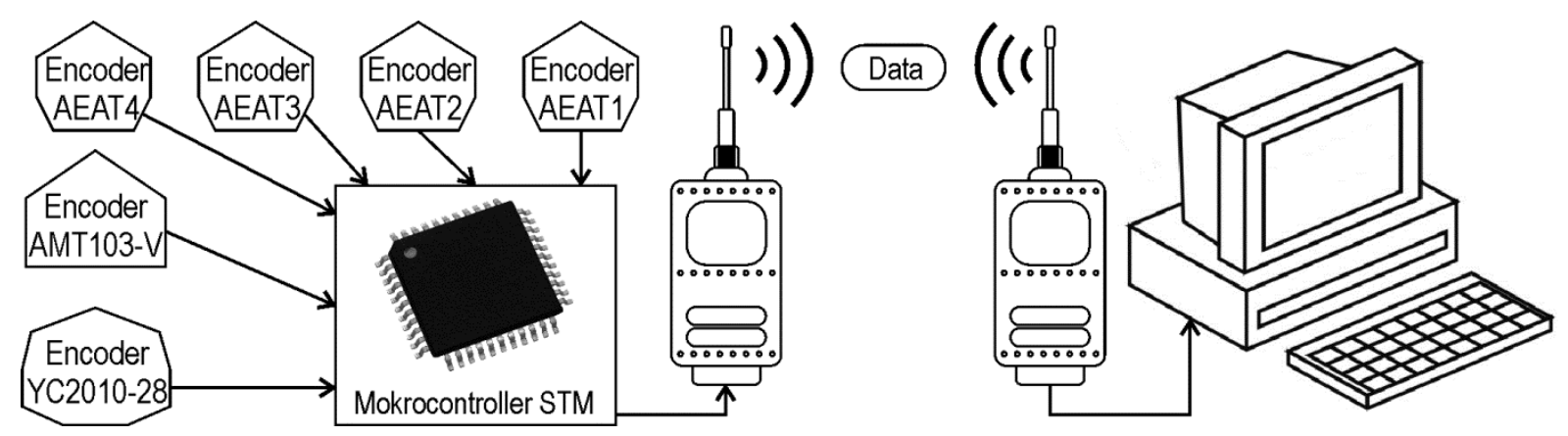

Fig. 3: Telemetry system scheme

\section{OpenGL implementation}

Based on the geometry of considered manipulator, its maximum operating range was calculated. In order to do so, Denavit-Hartenberg parameters were obtained and used to calculate transformation matrix of each joint. Further, those matrices allowed to calculate global transformation matrix. Based on it, it was very easy to calculate end effector's position as well as relative setup of each link based on data gathered from each encoder. (Yu, 2017).

Implementing the manipulator required creating a program in $\mathrm{C}++$ language using built-in functions from OpenGL library. OpenGL is open and free to use programming interface beneficial in making, e.g. application based on 3D graphics (Porebski, 2008). Written program will let us to easily control manipulator's movement even without line of sight view.

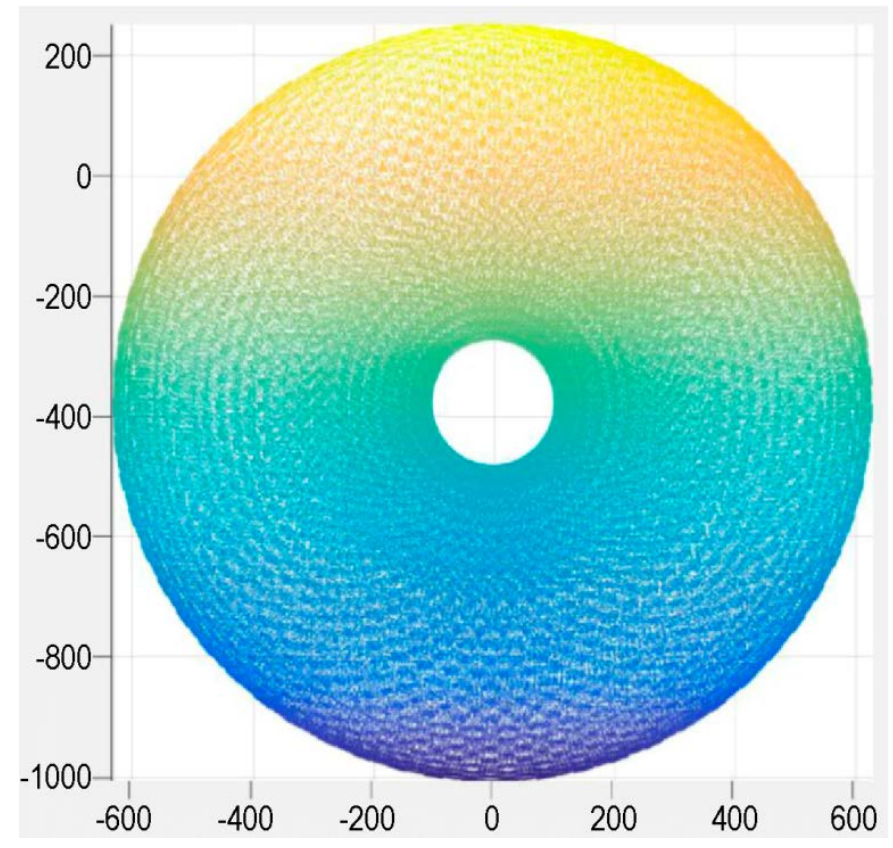

Fig. 4: The cloud of range points

After rigorous tests and fine-tuning of the system, it was ready for implementation to the rover's Command Centre Application. It is written in $\mathrm{C \#}$ programming language. The application is used for remote control of the Mars Rover Analogue and contains all critical functions needed for robot steering. Mars Rover analogues are used during the competition University Rover Challenge in the USA. The competition requires high manipulation ability from robot's arm during three of four tasks. Telemetry system during the competition may help with orienting the manipulator position at task field can be located even one and a half kilometre away from the operator. It is important to know the actual position of every joint of manipulator during tasks if the operator is not able to see robot by himself.

\section{Simulation and tests}

Calculated forward kinematics of the rover's arm as well as simulated one in the 3D environment lead to the comparison of the clouds of the range points. Both were obtained through simulation (in MatLab environment) of links movement within the range restricted by mechanical design. Each simulation step differed from previous one by 1 degree on each considered link. Further, generated cloud (Fig. 4) of end effector's points was compared and confirmed that both clouds were equal.

The last step was to test the performance of designed telemetry system. It was tested by two operators that did not participate in system's development process. They were asked to perform a pre-defined set of movements it two runs - one performed with the manipulator in visual range and second based only on visualisation provided by the system. Both operators expressed their approval to the systems performance. Each run's positioning error varied within $1 \mathrm{~cm}$ range. They did not find more it challenging to control 
robotic arm based only on its representation in 3D space. Their opinion on this project was positive and grants basis for further development.

\section{Conclusions}

Prior to the operation, the telemetry system has to be pre-configured. In case of incremental encoders, it is crucial to obtain useful information about relative position of each arm's link. The base measurement for the AMT103 encoder is taken when gripper's fingers are closed. YT2010 encoder' read is set to zero when

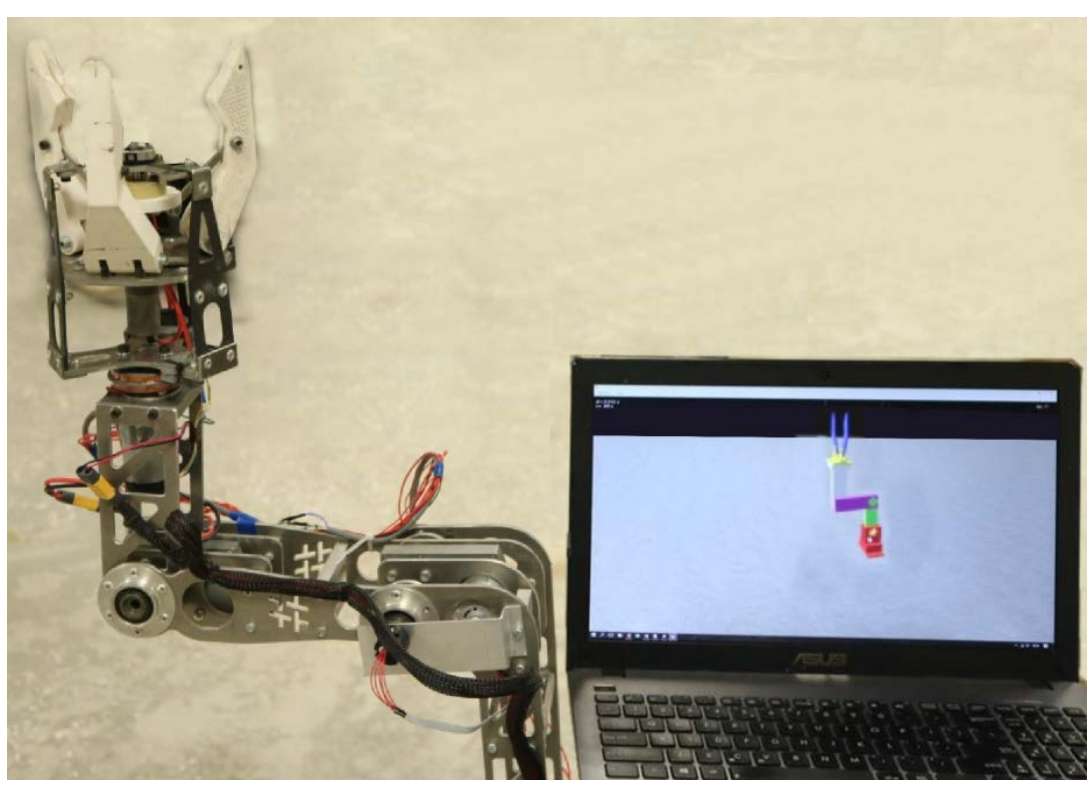

Fig. 5: Telemetry system one (selected) of the gripper's fingers is parallel to the arms plane.

Initial tests of the complete system indicated proper work within $200 \mathrm{~m}$ radius. It was caused by limited transmission speed due to use of a set of two radio modems in duplex mode. The range was extended by doubling the number of radio modems and switching to the simplex transmission. In this method, two lines are used. One to send and second to receive information between two nodes. This approach allowed to mitigate the range problem.

Telemetry system (Fig. 5) is a good solution to solve a problem with orientation assessment during the task out of the view field. With minimal effort, it may be ready to work with a variety of different manipulators. Usage of telemetry system should minimalise parts wear and damage due to wrong moves of the operator, that an on-board computer won't detect and avoid.

\section{References}

Craig, J.J. (1989) Introduction to robotics: Mechanics and control. Addison-Wesley, $2^{\text {nd }}$ edition.

Cruz, J.C., Garcia, R., \& Martinez-Ojeda, C.O. (2017) Biodiversity sustainability using telemetry for the remote transmission of data from Lake Buhi, Camarines Sur. 2017IEEE 9th International Conference on Humanoid, Nanotechnology, Information Technology, Communication and Control, Environment and Management (HNICEM), 1-5.

Dzierzek, K. (2009) Analysis of mechatronic position measuring systems. Bialystok University of Technology Press, Bialystok (in Polish).

Kozlowski, K. Dutkiewicz, P. Wroblewski, W. (2003) Modelling and control of robots. PWN Press, Warsaw (in Polish).

Porebski, W. (2008) C++ ISO in practice, PWN, Warsaw (in Polish).

SolidWorks Essentials. (2017) Dassault Systemes SolidWorks Corporation, Waltham.

Sydor, M. (2009) Introduction to CAD. Computer aided design for beginners, PWN, Warsaw (in Polish).

Yu, J. You, D. Liu, J. (2017) Analysis of inverse kinematics method for six degrees of freedom manipulator based on MATLAB. 2017 3rd IEEE International Conference on Control Science and Systems Engineering (ICCSSE) 211-215. 